Editorial

\title{
Instructions to Authors for Manuscript Submission
}

Kenneth A. Johnson ${ }^{1}$

\author{
${ }^{1}$ Sydney School of Veterinary Science, Faculty of Science, \\ University of Sydney, Sydney, New South Wales, Australia \\ Vet Comp Orthop Traumatol 2018;31:v.
}

In conjunction with the new page layout for Veterinary and Comparative Orthopaedics and Traumatology (VCOT), some changes have been made to our Instructions to Authors for manuscript submission. The new instructions were published in the previous issue of VCOT; they are also available online, on the VCOT home page at www.thieme-connect.com.

There are several points worthy of mention. For journal articles in your reference list, please list all authors' names, up to and including six names; for articles with more than six authors, list the only first three authors followed by et al.

Also, to improve the 'readability' of articles, the use of acronyms to abbreviate a name or term should be kept to an absolute minimum. All anatomical names and terminology

should be written in full without abbreviation. For example, cranial cruciate ligament, medio-lateral

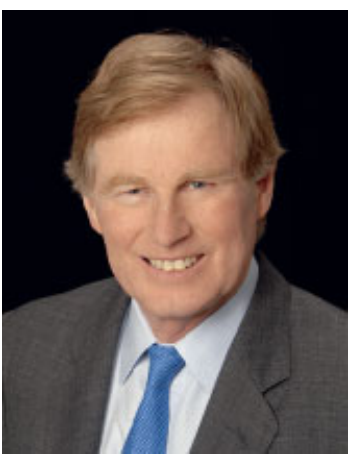

Kenneth A. Johnson view and total hip replacement are written in full.

Finally, authors of biomechanical studies are advised that the reviewers and editors will assess the strengths and weaknesses of the testing methodology. The significance of the results and the conclusions must be discussed in light of the limitations that are present. For the benefit of our authors, Dr. Simon Roe has provided more detailed guidelines about this matter in his Guest Editorial in this issue of the journal.

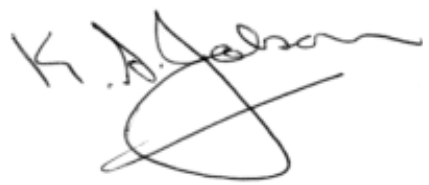

